\title{
Mesenchymal stromal cells protect from consequences of HSCT-transplantation preparatory irradiation: insights into possible mechanisms
}

\author{
${ }^{1}$ Claudia Lange, ${ }^{2}$ Rudolph Reimer, ${ }^{3}$ Jozef Zustin, ${ }^{1}$ Bärbel Brunswig-Spickenheier \\ ${ }^{1}$ Clinic for Stem Cell Transplantation, Dept. Cell and Gene Therapy, University Medical Center Hamburg-Eppendorf, \\ ${ }^{2}$ Technology Platform Microscopy \& Image Analysis Heinrich-Pette-Institut Hamburg, \\ ${ }^{3}$ Institute of Pathology, University Medical Center Hamburg-Eppendorf,
}

Priv.-Doz. Dr. rer. nat. Claudia Lange, Clinic for Stem Cell Transplantation, Dept. Cell and Gene Therapy, University Hospital Hamburg-Eppendorf, Martinistr. 52, 20246 Hamburg, Germany
Tel: $+49-40-741055917$

Fax: +49-40-7410 53034

E-mail: cllange@uke.de

\section{Abstract}

Ionizing irradiation is widely used as conditioning therapy in bone marrow (BM) transplantation. High-dose radiation treatment induces profound tissue damage, especially, of hematopoietic stem cells and progenitor cells. Efforts to improve clinical outcomes post- irradiation are focused on the hematopoietic stem cell niche. Mesenchymal stromal cells (MSCs) represent an integrative part of the $\mathrm{BM}$ stromal microenvironment. When co-transplanted with HSC, MSCs augment hematopoietic recovery after chemo- or radiotherapy. The aim of our study was to evaluate essential biological parameters of MSCs, with respect to their lineage-specific differentiation capacity, in vivo survival rates, as well as their ability to rescue lethally irradiated hosts. Materials and Methods. In vitro differentiation of human BM-derived MSCs (hMSCs) for hematopoietic (HSC) and endothelial cells (EC) was studied by reverse transcription-quantitative PCR (RT-qPCR) of lineage-specific surface markers and other proteins. To test in vivo ability of murine MSCs to rescue lethally irradiated (9.5 Gy) mice, the animals were transplanted with eGFP-marked murine MSCs (mMSCs). Long-term donor chimerism was assessed in blood, BM and thymus using CD45.2 and Y chromosome markers. A microarray analysis of bone marrow cells from MSC-transplanted animals was also performed, in order to compare their gene expression profiles to appropriate controls.

\section{Results}

Upon in vitro differentiation of hMSCs, the hematopoietically differentiated cells changed their gene expression towards a typical profile of progenitor and mature hematopoietic cells. A variety of transcription factors responsible for erythropoiesis, megakaryopoiesis, lympho- and myelopoiesis were up-regulated during differentiation in serum-containing media. A population of cells with small round or polymorphic nuclei was detected which expressed hematopoietic progenitor and mature antigen markers, albeit to a rather low degree. The same cells were able to acquire endothelial morphology and expressed endothelial genes upon cultivation with endothelial promoting factors. Following MSCs transplantation, the lethally irradiated mice showed normal hematopoietic recovery comparable to effects of HSC infusions. Seven months later, the recipients had normal distribution of peripheral blood cell populations. No evidence of donor chimerism was shown at any time 
point posttransplant. The distribution kinetics of eGFP+ donor cells after i.v. transplantation identified fast disappearance from peripheral blood, reaching ca. $2 \%$ donor mMSC in peripheral blood after 8 hours and significant entrapment in lungs, however, without long-term persistence and embolization events. The microarray expression trial in BM cells from MSC-treated animals has shown upregulation of the genes which are beneficial to $\mathrm{BM}$ reconstitution, whereas the genes with supposed radiation-related $\mathrm{BM}$ deterioration were downregulated. Injection of MSC-derived microvesicles to lethally irradiated animals provided similar protective effects, as transplantation of MSCs per se. Conclusion. Our results present another piece of evidence and possible mechanisms for highly effective paracrine mechanism working, e.g., upon BM populations, thus suggesting MSC-infusion to be an efficient treatment option following acute irradiation. Moreover, MSC transplantation might export their inherent trophic effect to unorthodox sites, e.g., the lung of a recipient.

\section{Keywords}

acute ionizing irradiation, hematopoietic recovery, mesenchymal stromal cells, protective effects

\section{Introduction}

Management of patients subjected to extensive body irradiation as a part of conditioning therapy still remains a major challenge. Survival of radiation-induced bone marrow failure depends on the dose of radiation received and the intensity of supportive care which can protect from otherwise lethal infection and give surviving stem cells a chance to replenish blood cell populations. Since radiation effects on blood stem cells occur at doses generally lower than those on other critical organs, the rapidly emerging changes in the peripheral blood cell lineages determine the treatment options. In fact, total body irradiation (TBI) at doses more than 7-8 Gy in humans corresponds to medullar eradication. Under this threshold, spontaneous recovery from residual hematopoietic stem and progenitor cells may be expected within 30-50 days, however, preceded by cytopenic phases of granulocytic, megakaryocytic and erythrocytic lineages. Interestingly, even after TBI, intrinsically radioresistant stem cells have been detected in distinct bone marrow (BM) areas comprising a residual hematopoietic stem and progenitor cell pool [10]. Acute irradiation does not only imply damage to the bone marrow. In a dose-dependent matter, it can also emerge as gastrointestinal and cerebrovascular syndromes leading to development of multiple organ dysfunction (1). Damage to the whole organism is related to a systemic inflammatory response. Different target organs are affected due to activation of innate immune system, resulting in a significant release of inflammatory cytokines [4]. The pathophysiology of such tissue damage appears comparable to that of acute graftversus-host disease (GvHD) following allogeneic stem cell transplantation where a similar "cytokine storm" has been observed [6]. In absence of appropriate treatment, oxidative stress after high dose ionizing radiation has been involved in delayed morbidity [4]. Management of acute radiation syndrome relies, therefore, on tissue damage repair processes that might be supported by therapies aimed for mitigation of inflammation [4].

Efforts to improve outcome after irradiation focus on the stem cell niche. Therefore, prospective therapies should augment the hematopoietic niche activity to accelerate the in vivo recovery of blood cell populations. Several studies have demonstrated that BM osteoblasts regulate the HSC pool size in vivo via the Jagged1-Notch signaling pathway [7]. For example, parathyroid hormone receptor activation can increase the number of osteoblastic cells, thus resulting in Notch1-mediated expansion of HSC [2]. Mesenchymal stromal cells (MSC) comprise an integrative part of the BM stroma, being also described as osteoblastic progenitors [8]. MSC are multipotential nonhematopoietic progenitor cells capable of differentiating into multiple lineages of the mesenchyme. In bone marrow, the local stromal cells surround HSC and their progeny. The hematopoietic niche provides a sheltering microenvironment that provides maintenance and self-renewal of HSC by shielding them from differentiation and apoptotic stimuli that would otherwise challenge stem cell reserves. Moreover, the hematopoietic niche also controls proliferation and differentiation of HSC and release of mature progeny into peripheral blood flow. Regulation of HSC quiescence, by maintenance of resting HSC in endosteal niche, control of HSC proliferation, differentiation and recruitment in the vascular niche can be ascribed to bone-marrow stromal cells [27]. Thus, physiological role of MSCs is not a mere replacement of mesenchymal tissues such as bone. Moreover, their primary and most important function is to inhibit immunosurveillance and to establish a protective and regenerative microenvironment for HSC.

Clinically, MSCs have been proven to intervene with acute organ impairment. When co-transplanted with HSC, MSCs augment hematopoietic recovery after chemo- or radiotherapy significantly decreasing the time to full hematopoietic and particularly platelet reconstitution [12]. Additionally, there is evidence for MSC effectiveness in the treatment of steroid resistant GvHD without any side effects, even when obtained from BM of third-party donors [18]. No HLA-match is needed between donor and recipient because MSCs have been shown to be hypoimmunogenic and are not recognized by the recipient immune system even after repeated injections [18]. Finally, MSCs secrete a variety of bioactive molecules [22]. Among those, some essential hematopoietic growth factors including IL-6, IL-11, leukemia inhibitory factor (LIF), stem cell factor (SCF) and Flt3 ligand are produced, as well as factors with immunomodulatory effects, e.g. transforming growth factor- $\beta 1$ (TGF- $\beta 1$ ), prostaglandin E2, indoleamine 2,3-dioxygenase, and others [21]. Additionally, vascular endothelial growth factor (VEGF) secreted by MSCs in abundance might interfere with 
early apoptotic cell death after irradiation [10]. Therefore, MSCs might be a good candidate for modulation of the hematopoietic niche activity.

In summary, MSCs have emerged as a promising therapeutic tool for tissue regeneration and repair. Further clinical interest has been raised by the observation that MSCs are immunoprivileged and might be transplanted from unrelated, i.e. allogeneic donors [21,27]. Altogether, we assumed that MSCs, with their comprehensive trophic potential, could serve as a readily available treatment option after severe radiation exposure. The aim of our study was to evaluate essential biological parameters of MSC, with respect to their lineage-specific differentiation capacity, in vivo survival rates, as well as their ability to rescue lethally irradiated hosts.

\section{Methods and Results}

\section{In vitro differentiation of human MSC (hMSC)}

As first experiments, we investigated the capability of human BM-derived MSCs (hMSCs) to differentiate into progenitors for hematopoietic (HSC) and endothelial cells (EC). The hu- man MSCs were thoroughly characterized according to the ISCT (International Society for Cellular Therapy) criteria [5], including flow cytometry and their capability to differentiate into three mesodermal lineages [16]. To avoid any contamination of MSCs with HSC, cloned cells were used exclusively. Cloned human MSCs were subjected to differentiation into (i) hematopoietic cells using serum-containing or serum-depleted growth conditions and (ii) endothelial cells (for technical details see ref. 14). Fibroblastoid MSCs (Fig. 1a) formed blast-like cells with noticeably decreased diameter from originally $28.9 \pm 6.6$ to $15.7 \pm 3.5 \mu \mathrm{m}$ during the differentiation into hematopoietic (Fig. 1b) and endothelial (Fig. 1c) lineages. The in vitro conditions led to cluster formation appearing as an in vitro equivalent of stromal structures from which differentiation proceeded. The cells committed to hematopoietic lineage changed their gene expression towards appropriate profiles of blood cell progenitors (CD117, CD133, CD45) and mature (CD14, CD16, glycophorinA GlyA, CD31, podoplanin PDPN) hematopoietic cells (Fig. 2a). Interestingly, the erythropoietin receptor (EPOR) was upregulated in almost all clones and under all conditions suggesting a definite role for EPO in proliferation and differentiation of mesodermal progenitors. Additionally,
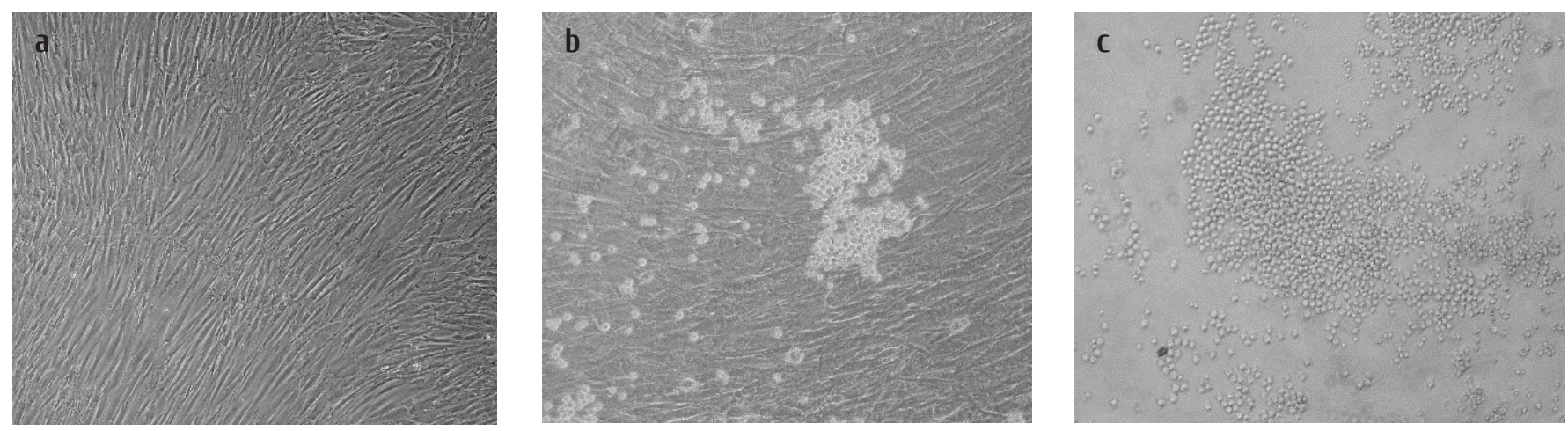

Figure 1: Human MSC display a fibroblastoid morphology during in vitro expansion but form blast-like cells after induction of differentiation. One clonal hMSC culture is shown during expansion (a), differentiation into hematopoietic (b) or endothelial (c) cells.

a

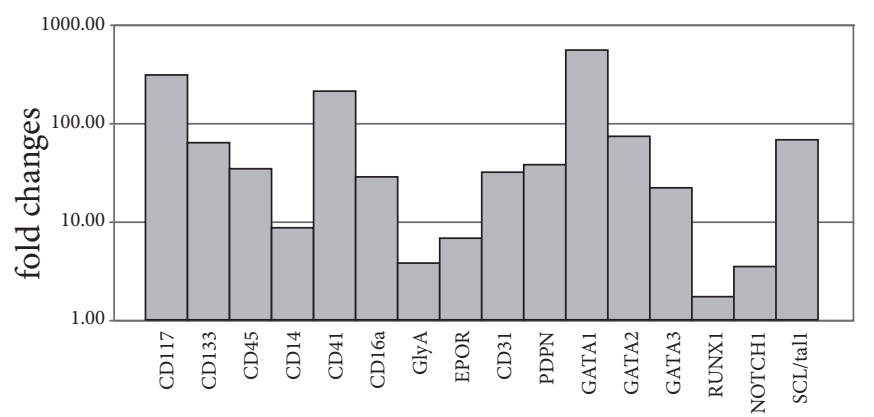

b

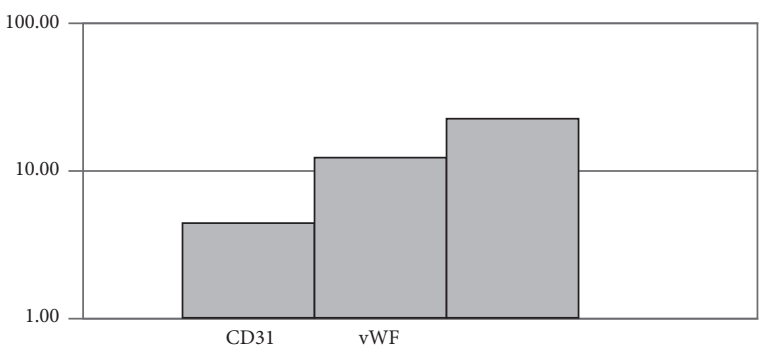

Figure 2: Human MSC significantly upregulate expressions of hematopoietic and endothelial genes after induction of differentiation. Shown are the fold changes of gene expressions of indicated hematopoietic (a) and endothelial (b) genes after differentiation compared to undifferentiated hMSC. GlyA, Glycophorin A; vWF, von Willebrand factor; VEGFR, vascular endothelial growth factor receptor. 
a variety of transcription factors responsible for erythropoiesis (SCL/tal1), erythro-megakaryopoiesis (GATA1, GATA2), lymphopoiesis (GATA3), and myelopoiesis (NOTCH1, RUNX1) were upregulated upon serum-containing differentiation. As SCL and RUNX1 are transcription factors essential for HSC formation by instructing lineage specification (9), we suggested an efficient induction of this differentiation pathway in MSCs. Using immunofluorescence, a subpopulation of antigen-positive cells with small round or polymorphic nuclei was detected, showing expression of hematopoietic progenitor and mature antigen expression (not shown, refer to ref. 14), albeit to a rather low degree. In parallel, the same cells were able to acquire endothelial morphology and expressed endothelial genes upon cultivation with endothelial promoting factors (Fig. 2b). At the protein level, single double positive cells for CD31/vWF (von Willebrand factor) and VEGFR-2/CD34 were detected [14].

Hematopoietic and endothelial progenitors share expression of a number of genes, including VEGFR-2, CD34, SCL, GATA2, RUNX1, and CD31, suggesting that investigated hMSCs possess in vitro hemangioblastic capacity, and might act as extrinsic differentiation factors and lineage-inducing regulators. Most potent differentiation was achieved in cultures where the majority of hMSCs adopted stromal function, thus inducing a minor part for differentiation.

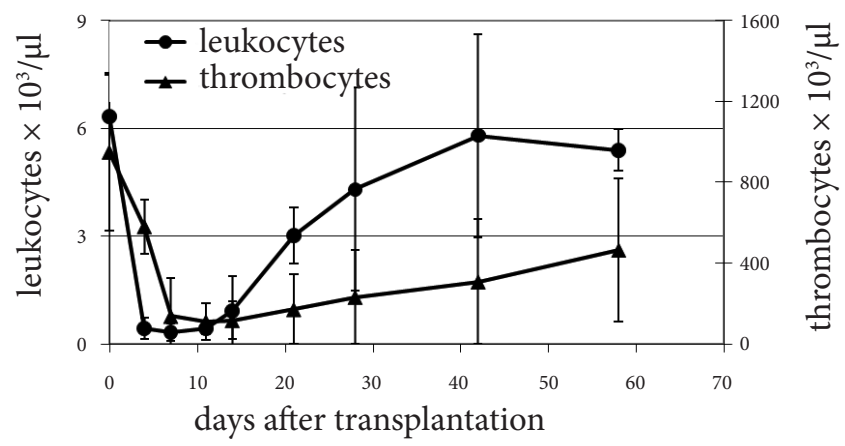

We concluded from the in vitro results, that MSCs might reconstitute the hematopoietic system. Hypothetically, one pluripotent stem cell would suffice to rescue lethally irradiated hosts. In reality, however, approx. 6 cells are needed [13], i.e. six pluripotent MSCs with the respective potential might suffice to restore hematopoiesis in vivo.

\section{MSCs promote hematopoietic recovery after lethal irradiation}

To test in vivo ability of murine MSCs to replenish the hematopoiesis after eradication, lethally irradiated (9.5 Gy) female recipients of the $\mathrm{C} 57 \mathrm{Bl} / 6 \mathrm{~J}-\mathrm{CD} 45.1$ strain were subjected to i.v. transplantation with $10^{6}$ eGFP-marked male bulk-culture C57Bl/6J mouse MSCs (mMSCs). Mouse MSC were cultured in DMEM/Ham's F12 + 20\% preselected FCS + Glutamin + ß-mercaptoethanol and cells after 9-12 passages used for transplantation. Leukocyte and thrombocyte recovery was similar to recipients transplanted with HSCs (Fig. 3) reaching normalization of white blood cell counts after 4 weeks. Seven months later, the recipients were hematologically well, with a normal distribution of peripheral cell populations (Table 1). Similar experiments were carried out with clonal mMSCs showing one clone (IXH8) with superior survival promoting properties (Table 2). Noteworthy, the IXH8 clone was different from all other cultures showing long-stretched morphology and increased CD34 and CD45, however, without CD105 expression (Table 2).

Figure 3: Mouse MSC rescue mice after total body irradiation. Transplantation of bulk mMSC led to a normalization of the peripheral white blood cell count within 4 weeks. Thrombocyte recovery needed approx. 8 weeks for normalization.

Table 1: Peripheral blood cell populations in mMSC transplanted animals. Shown is the distribution of white blood cells 5 months after bulk mMSC transplantation estimated using Pappenheim-stained blood smears.

\begin{tabular}{|l|l|l|l|}
\hline Lymphocytes & Neutrophils & Monocytes & Eosinophils \\
\hline $72 \% \pm 3$ & $21 \% \pm 3$ & $5 \% \pm 2$ & $2 \% \pm 1$ \\
\hline
\end{tabular}


Table 2: Phenotypical characterization of mMSC and recipients' survival rates after transplantation.

Cultures of eGFP-transduced bulk and cloned mMSC after extended expansion were positive for CD59, CD105 and Sca-1 but negative for the hematopoietic markers CD34, CD45, CD117 and for CD90 by flow cytometry. Clone IXH8 was different from all other cultures in its expression of CD34/CD45 and negativity of CD105 (shown in bold italic). Transplantation with this clone resulted in the highest survival rate of the irradiated recipients, suggesting elevated CD34 and CD45 and no CD105 expressions might be a prerequisite of the high rescue capability. nd, not done.

\begin{tabular}{|l|l|l|l|l|l|l|l|l|}
\hline & CD34 & CD45 & CD59 & CD90 & CD105 & CD117 & Sca-1 & survival at 7 months [\%] \\
\hline bulk & 1.6 & 0.5 & 95.4 & 0.5 & 85.9 & 0.9 & 96.7 & $\begin{array}{l}19 / 28 \\
{[67.9]}\end{array}$ \\
\hline IXH8 & 9.8 & 4.1 & 97.4 & 2.7 & 1.6 & 1.5 & 99.2 & $\begin{array}{l}15 / 17 \\
{[88.2]}\end{array}$ \\
\hline IVH7 & 1.2 & 1.3 & 54.7 & 0.5 & 94.1 & 2.8 & 81.9 & $\begin{array}{l}2 / 12 \\
{[16.7]}\end{array}$ \\
\hline IXC2 & 0.9 & 2.2 & 79.6 & 1.2 & 94.0 & 1.5 & 90.2 & $\begin{array}{l}3 / 10 \\
{[30]}\end{array}$ \\
\hline VIIIE7 & 1.2 & 1.1 & 71.1 & 2.0 & 93.1 & 1.5 & 96.4 & $\begin{array}{l}4 / 10 \\
{[40]}\end{array}$ \\
\hline VF10 & 2.2 & 2.2 & 45.9 & 0.7 & 74.0 & 3.4 & 77.9 & $\begin{array}{l}3 / 10 \\
{[30]}\end{array}$ \\
\hline radiation control & nd & nd & nd & nd & nd & nd & nd & $\begin{array}{l}0 / 15 \\
{[0]}\end{array}$ \\
\hline
\end{tabular}

\section{Transplanted donor cells are detectable} short- but not long-term

To trace donor chimerism in recipients, we stained recipient peripheral blood (PB), BM and thymus cells with CD45.2 antibodies and carried out flow cytometry. Interestingly, no CD45.2-positive cells were found at any time point, thus not showing regeneration through donor cells.
Y-chromosome-based chimerism analysis in female recipients using specific Y-chromosome primers for quantitative PCR could not detect donor cells in any of investigated tissues including PB and BM (not shown), although animals survived up to the final evaluation after 7 months. Spectral karyotyping of clonal mMSC revealed loss of Y-chromosome (Fig. 4), whereas bulk cultures were still Y-positive at passage13 (not shown).

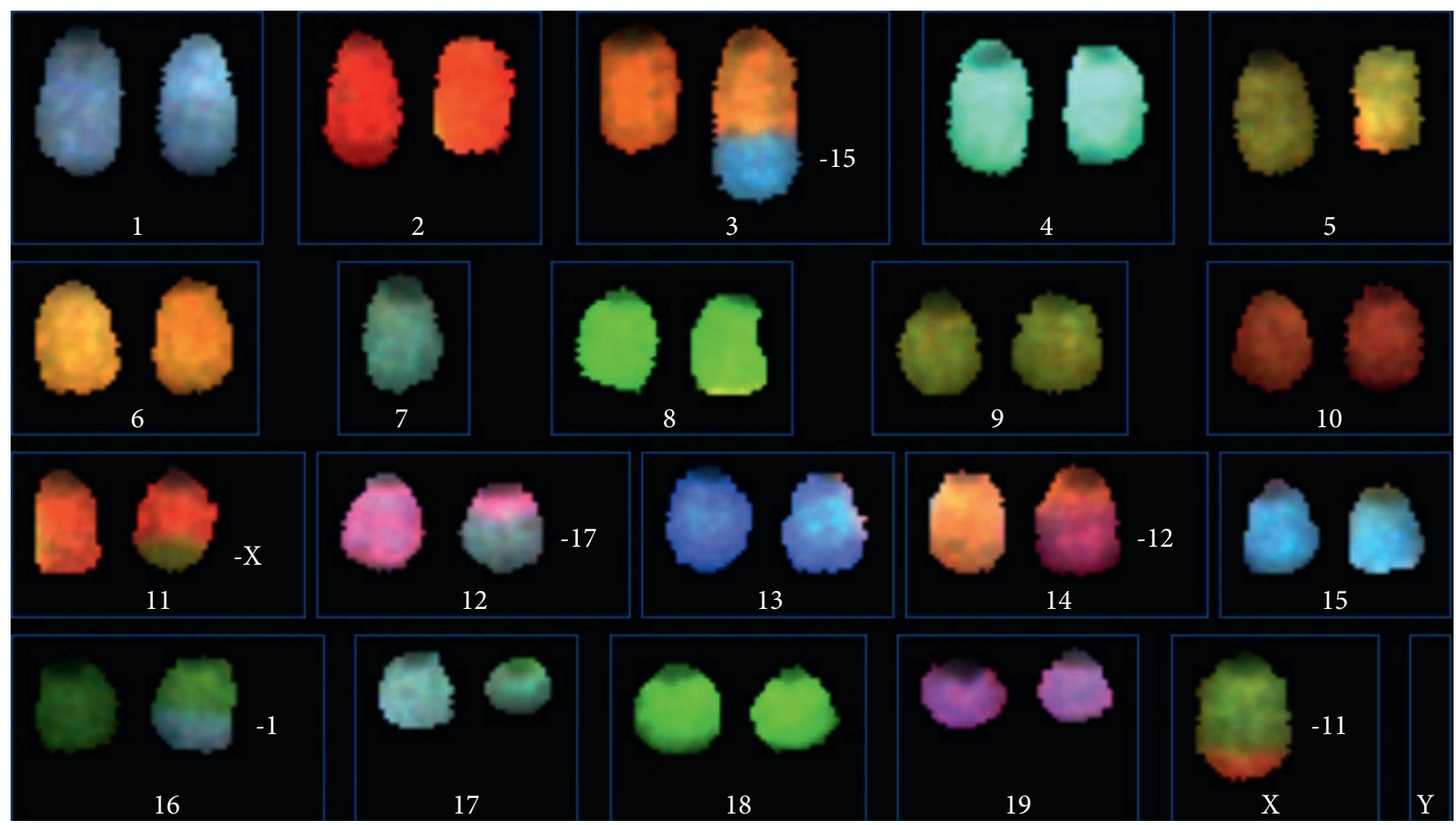

Figure 4: Spectral karyotyping of mMSC. Shown is the SKY analysis of clone IXH8. SKY analysis of a representative diploid metaphase revealed the loss of the Y-chromosome and this has been observed in all metaphases analyzed. 
Next, we used eGFP-specific primers for quantitative PCRbased donor cell detection. Primers for stably integrated eGFP-sequences, however, also failed to detect any donor cells, and no eGFP-positive cells were found in blood, BM or thymus by flow cytometry. Although we cannot completely rule out single donor cells below the detection limit, hematopoietic recovery in recipients is unlikely due to replacement with donor cells. This conclusion contradicts earlier results of hematopoietic recovery after myeloablative TBI with blood-derived mMSCs $[11,15]$ showing donor characteristics in blood and BM. One fundamental difference between both cell sources is potential in vitro immortalization, altering BM seeding capability of MSC. Therefore, our results support the concept of impaired transplantability of expanded MSC [24] but also challenge the hypothesis of high plasticity of MSC [1].
The distribution kinetics of eGFP+ donor cells after i.v. transplantation identified fast disappearance from $\mathrm{PB}$, reaching ca. $2 \%$ after 8 hours and no cells at d10 (Fig. 5a). In contrast, mMSC trapped in lungs quickly (Fig. 5b), however without long-term residence and embolization as shown by lack of donor signals after $\mathrm{d}+10$. Accordingly, no donor cells were detectable evident in the spleen, liver, BM (Fig. 5b), aorta, kidney, intestine, fat, thymus or lymph nodes (not shown). Although we did not find donor derived MSC in the BM, the morphology of this organ was preserved by MSC transplantation showing a normal distribution between different compartments (Fig. 6). Without MSCs, adipocytes are shown to dominate within short time, thus destroying the marrow structure. a

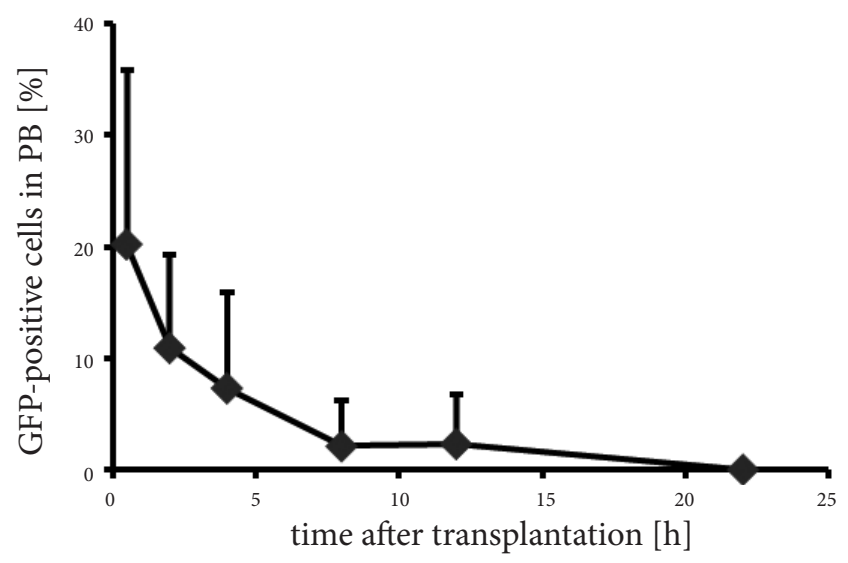

b

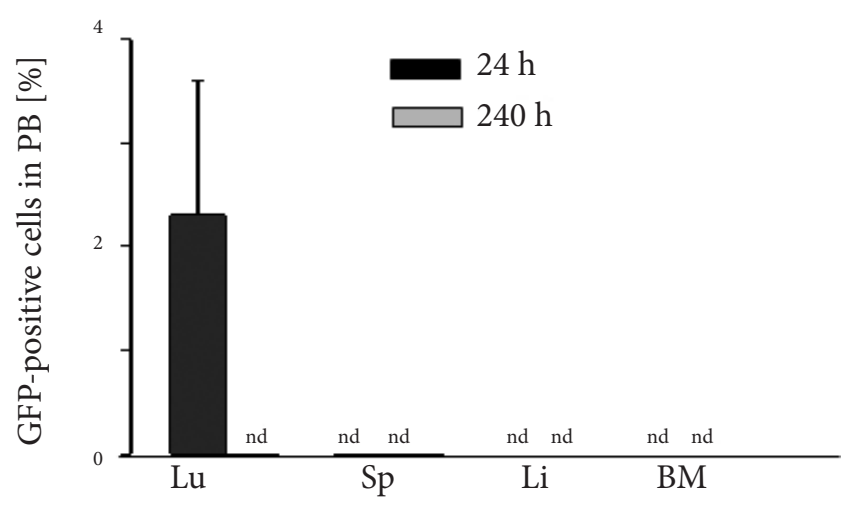

Figure 5: Donor mMSC are not detectable at longer terms.

(a) Tracking of eGFP-labeled clonal IXH8 donor mMSC after transplantation revealed a fast decrease in peripheral blood (PB). Within 8 hours, approx. $2 \%$ were quantified in $\mathrm{PB}$ and none after 10 days ( $\mathrm{n}=8$ for each time point). (b) mMSC accumulated in lungs (Lu) within $24 \mathrm{~h}$ and disappeared within 10 days $(240 \mathrm{~h})$. Spleen (Sp), liver ( $\mathrm{Li})$ and BM were negative at $\mathrm{d} 1$ and d10. nd, not detected.
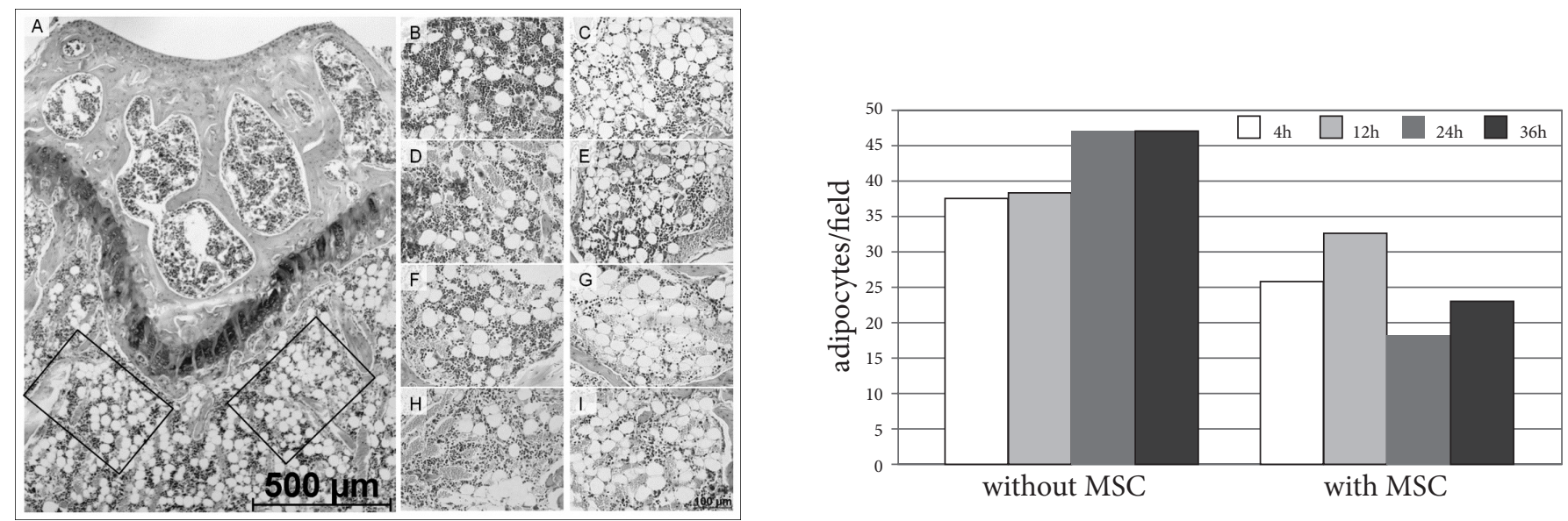

Figure 6. Histomorphology of BM with and without MSC transplantation.

Paraffin embedded long bones from MSC-transplanted or control animals were cut and the number of adipocytes counted in 2 designated areas (A) per bone from mice with MSC transplantation after 4 (B), 12 (D), 24 (F) and 36 (H) hours or without MSC transplantation (C, E, G, and I respectively). The lower figure shows the number of adipocytes at each time point. 


\section{MSCs change the BM gene expression}

While donor mMSC did not home to the BM, we observed a long term recipients' survival and assumed an influence of MSCs on the BM function. Therefore we carried out microarray analysis of bone marrow cells from MSC-transplanted animals, and compared their gene expression profiles to that of HSC-transplanted animals and age-matched controls [14]. The gene expression profile in BM changed significantly, clustering into separate group as compared to untreated $\mathrm{BM}$ or HSC-transplanted mice. Validation of selected genes with high variance proved a beneficial role of MSC in endogenous hematopoietic reconstitution. MSCs caused upregu-

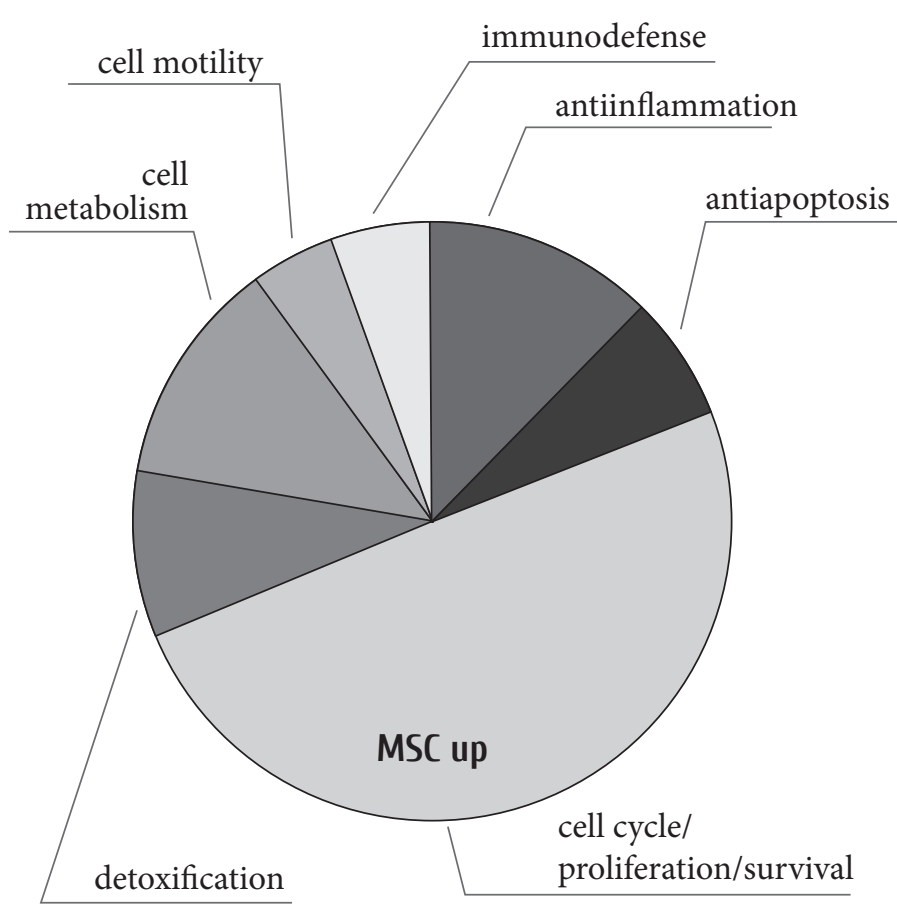

lated protection from oxidative stress, cell cycle, anti-inflammatory and detoxication events (e.g. BRPK, Cdkn1a, Thbs2, Gstm5 gene expression) in a complex way, along with downregulation of lymphoid development, pro-inflammatory events, protein degradation and adhesion/matrix formation for improved cell motility (e.g. gene expressions of Vpreb1, Rag2, Klk6, Klk1b5, Uchl1, Sykb, Gpam, Col5a3, Emid1) [14]. Upon summarising the microarray expression data, we have shown upregulation of the genes which are beneficial to $\mathrm{BM}$ reconstitution, whereas the genes with supposed radiation-related BM deterioration were downregulated (Fig. 7).

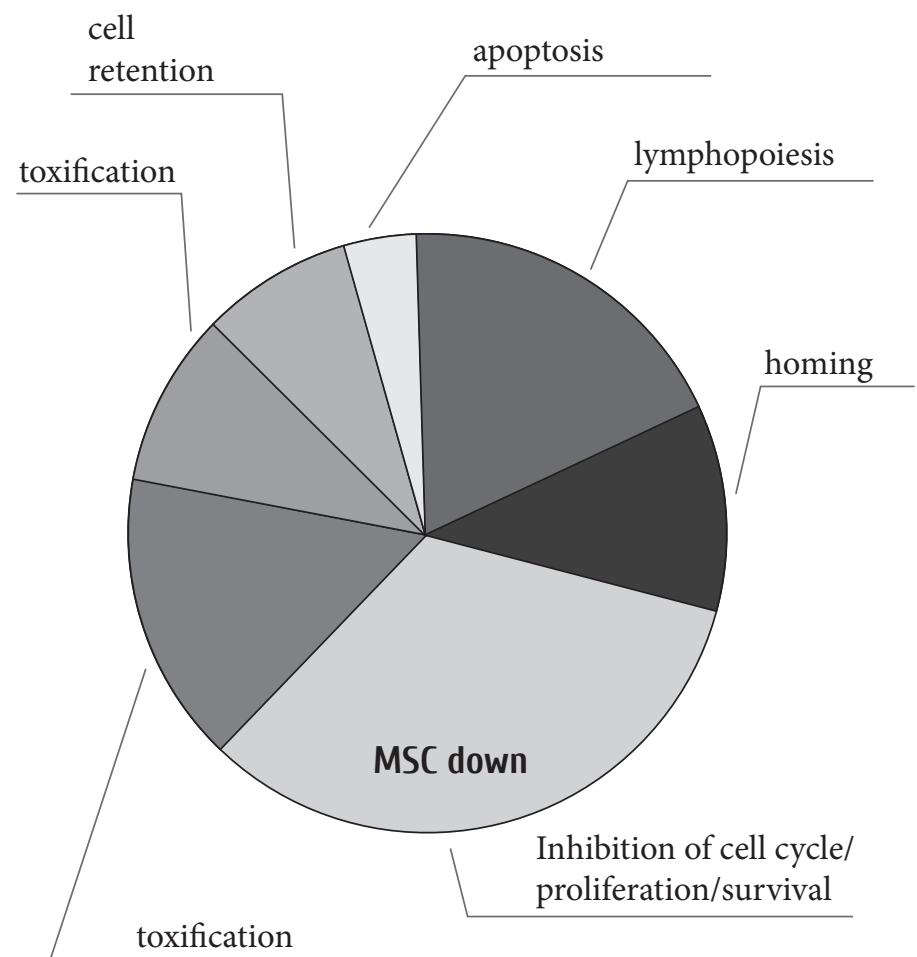

Figure 7. MSC transplantation into lethally irradiated animals changes the gene expression in the bone marrow. Gene expression data were generated using microarray analysis and significantly regulated genes clustered into functional groups. Shown are upregulated functional gene groups (MSC up) or downregulated (MSC down) in MSC-transplanted animals.

\section{Potential paracrine mechanism of MSC}

Potential mechanisms mediating bone marrow protection by MSCs entrapped in the lung, still remain unclear. Recently, we could show that injection of MSC-derived microvesicles to lethally irradiated animals provided similar protective effects, as transplantation of MSCs per se (Fig. 8). The microvesicles represent a fraction of ultra-small lipid bilayer particles of 30 to $1000 \mathrm{~nm}$ size (including exosome fraction) which are known to shuttle proteins, lipids, mRNA and microRNA [25]. Any of these components could participate in radiation protection and recovery of the bone marrow. Interestingly, the microvesicle-associated reconstitution of platelet scores occurred at a faster time frames, as compared to MSCs injections. Further work should reveal a more precise mechanism conferring radiation protection associated with MSC microvesicles.

\section{Discussion}

In this study we present an evidence that donor MSCs do not directly reconstitute the hematopoietic system following radiation insult. However, these cells may provide salvage for the surviving HSCs. Acute irradiation produces excessive inflammatory responses (23) which contribute to HSC death if untreated. Along with other organs, the lung is also heavily affected by radiation damage and might retard MSCs. Mesenchymal cells interfere with inflammation by changing overall gene expression profile, both in lungs where they are captured, and in bone marrow compartments. Assuming this, a direct MSC homing to the bone marrow is not necessary for changed gene expression patterns. This mechanism has been described in murine model of myocardial infarction where hMSCs have been shown to produce antiapoptotic TSG6 without significant engraftment [19]. A paracrine, differentiation-independent effect of MSCs did also ameliorate kidney injury $[17,26]$. 


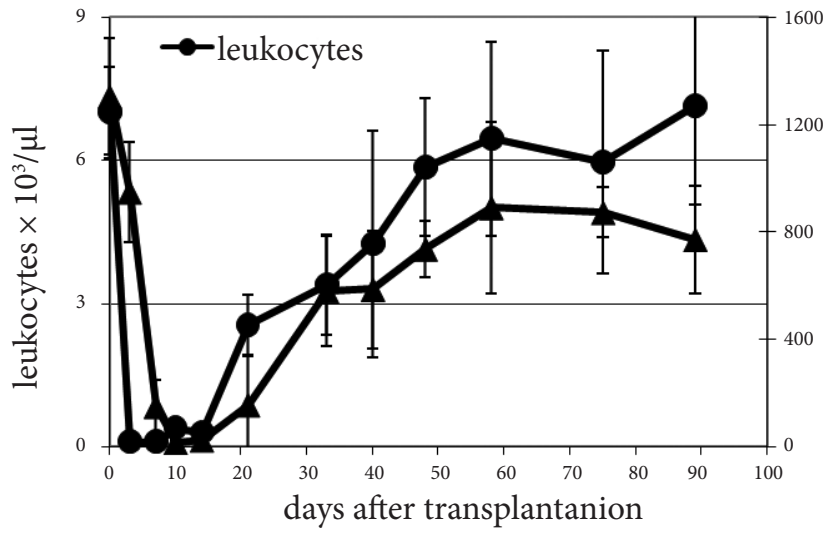

b

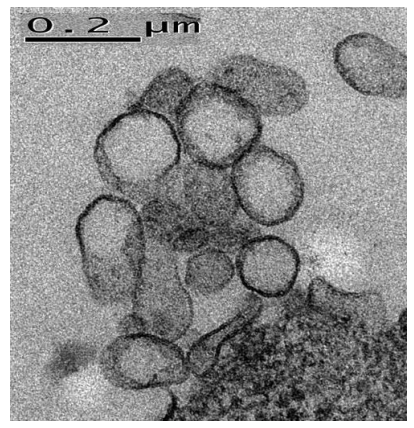

C

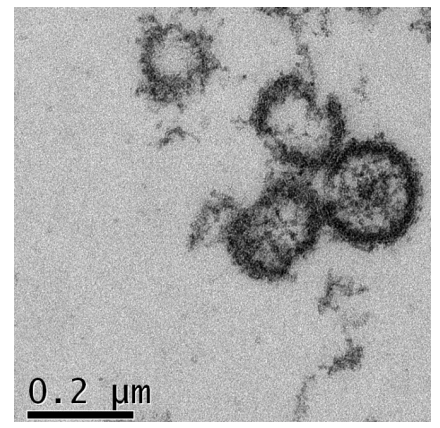

Figure 8: Transplantation of mMSC-derived microvesicles rescues lethally irradiated animals.

Leukocyte counts after mMSC-derived microvesicle injection normalized with similar kinetics as with mMSC, whereas thrombocyte counts showed a much faster normalization $(\mathrm{a})(\mathrm{n}=15)$. Electron microscopy of microvesicles released from mMSC $(\mathrm{b})$ and purified by ultracentrifugation (c).

What could be expected from MSC as a potential therapeutic tool? Secretion of broad-range bioactive molecules is now believed to be the main mechanism by which the therapeutic effects of MSCs are achieved [20]. MSCs may secrete active factors that (a) inhibit apoptosis and limit the extent of cellular damage; (b) inhibit fibrosis or scarring at the injured sites; (c) protect microvasculature and stimulate angiogenesis, thus improving perfusion rates; and (d) promote proliferation of tissue-specific progenitor cells, as shown for cardiac-, neural- and kidney-specific stem cells $[26,27]$. In parallel, we have shown in a model with acute irradiation that MSCs boosted anti-inflammatory, anti-apoptotic, detoxifying, cell cycle and anti-oxidative stress control, whereas proinflammatory effects, extracellular matrix formation, and adhesion properties were decreased. In general, MSC injections may result into systemic improvements counteracting deleterious effects of myelosuppression [14].

In conclusion, transplanted MSC might export their inherent trophic effect to unorthodox sites [3], e.g. to lungs. Our results present another piece of evidence for this highly effective paracrine mechanism which may work, e.g., in BM populations, suggesting MSC-infusion to be an efficient treatment option following acute irradiation. Despite some limitations in our existing knowledge, a capacity of MSCs, or MSC-derived microvesicles, to exert hematopoietic support via a bystander mechanisms, might indicate that persistent engraftment at the site of damage is not a mandatory prerequisite. Importantly, a very short-term residence of MSCs in lung and/or the entire organism might critically contribute to the safety of this cell-based therapy, by avoiding potential side effects as tumor formation or maldifferentiation.

\section{Acknowledgements}

There are no commercial associations that might create a conflict of interest in connection with this paper.

This work was supported by the Federal Ministry of Education and Research, Germany, grant number 13N8904 and by the "Deutsche José Carreras Leukämie-Stiftung e.V.", grant number DJCLS R 12/30.

\section{References}

1. Anderson DJ, Gage FH, Weissman IL. Can stem cells cross lineage boundaries? Nature Med 2001; 7:393-395.

2. Calvi LM, Adams GB, Weibrecht KW, Weber JM, Olson DP et al. Osteoblastic cells regulate the haematopoietic stem cell niche. Nature 2003; 23: 841-846.

3. Caplan AI, Dennis JE. Mesenchymal stem cells as trophic mediators. J Cell Biochem 2006; 98: 1076-1084.

4. Chao NJ. Accidental or intentional exposure to ionizing radiation: biodosimetry and treatment options. Exp Hematol 2007; 35: 24-27.

5. Dominici, M. et al. Minimal criteria for defining multipotent mesenchymal stromal cells. The International Society for Cellular Therapy position statement. Cytotherapy 2006; 8:315-317.

6. Ferrara JLM, Levy R, Chao NJ. Pathophysiologic mechanisms of acute graft-vs.-host disease. Biol Blood Marrow Transplant 1999; 5:347-356.

7. Fliedner TM, Chao NJ, Bader JL, Boettger A, Case C Jr et al. Stem cells, multiorgan failure in radiation emergency medical preparedness: a U.S./European Consultation Workshop. Stem Cells 2009; 27:1205-1211.

8. Friedenstein AJ, Chailakhyan RK, Latsinik NV, Panasyuk AF, Keiliss-Borok IV. Stromal cells responsible for transferring the microenvironment of the hemopoietic tissues. Cloning in vitro and retransplantation in vivo. Transplantation 1974; 17: 331-340.

9. Graf T, Enver T. Forcing cells to change lineages. Nature 2009, 462:587-594.

10. Hérodin F, Drouet M. Cytokine-based treatment of accidentally irradiated victims and new approaches. Exp Hematol 2005; 33:1071-1080.

11. Huss R, Lange C, Weissinger EM, Kolb HJ, Thalmeier K. Evidence of peripheral blood derived, plasticadherent 
CD34(-/low) hematopoietic stem cell clones with mesenchymal stem cell characteristics. Stem Cells 2000; 18:252-260.

12. Koç ON, Gerson SL, Cooper BW, Dyhouse SM, Haynesworth SE et al. Rapid hematopoietic recovery after coinfusion of autologous-blood stem cells and culture-expanded marrow mesenchymal stem cells in advanced breast cancer patients receiving high-dose chemotherapy. J Clin Oncol 2000; 18:307-316.

13. Krause DS, Theise ND, Collector MI, Henegariu O, Hwang S et al. Multi-organ, multilineage engraftment by a single bone marrow-derived stem cell. Cell 2001; 105:369-377.

14. Lange C, Brunswig-Spickenheier B, Cappallo-Obermann H, Eggert K, Gehling UM et al. Radiation rescue: mesenchymal stromal cells protect from lethal irradiation. PLoS One 2011; 5: 6(1):e14486. doi: 10.1371/journal.pone.0014486.

15. Lange C, Kaltz C, Thalmeier K, Kolb HJ, Huss R. Hematopoietic reconstitution of syngeneic mice with a peripheral blood-derived, monoclonal CD34-, Sca-1+, Thy-1(low), c-kit+ stem cell line. J Hematother Stem Cell Res 1999; 8:335-342.

16. Lange C, Schroeder J, Lioznov MV, Zander AR. High-potential human mesenchymal stem cells. Stem Cells Dev 2005; 14:70-80.

17. Lange C, Tögel F, Ittrich H, Clayton F, Nolte-Ernsting C, Zander AR, Westenfelder C. Administered mesenchymal stem cells are renoprotective in ischemia/reperfusion acute renal failures in rats. Kidney Int 2005; 68:1613-1617.

18. Le Blanc K, Frassoni F, Ball L, Locatelli F, Roelofs H et al. Developmental Committee of the European Group for Blood and Marrow Transplantation. Mesenchymal stem cells for treatment of steroid-resistant, severe, acute graft-versushost disease: a phase II study. Lancet 2008; 371:1579-1586.
19. Lee RH, Pulin AA, Seo MJ, Kota DJ, Ylostalo J et al. Intravenous hMSC improve myocardial infarction in mice because cells embolized in lung are activated to secrete the anti-inflammatory protein TSG-6. Cell Stem Cell 2009; 5:54-63.

20. Meirelles L da S, Fontes AM, Covas DT, Caplan AI. Mechanisms involved in the therapeutic properties of mesenchymal stem cells. Cytokine Growth Factor Rev 2009; 20:419-427.

21. Nauta AJ, Fibbe WE. Immunomodulatory properties of mesenchymal stromal cells. Blood 2007; 110:3499-3506.

22. Phinney DG, Prockop DJ. Concise review: mesenchymal stem/ multipotent stromal cells: the state of transdifferentiation and modes of tissue repair current views. Stem Cells 2007; 25:2896-2902.

23. Remberger M, Sundberg B. Cytokine production during myeloablative and reduced intensity therapy before allogeneic stem cell transplantation. Haematologica 2004; 89:710716.

24. Rombouts WJ, Ploemacher RE. Primary murine MSC show highly efficient homing to the bone marrow but lose homing ability following culture. Leukemia 2003; 17:160-170.

25. Théry C, Ostrowski M, Segura E. Membrane vesicles as conveyors of immune responses. Nat Rev Immunol 2009; 9:581-593.

26. Tögel F, Hu Z, Weiss $\mathrm{K}$, Isaac J, Lange C, Westenfelder C. Administered mesenchymal stem cells protect against ischemic acute renal failure through differentiation-independent mechanisms. Am J Physiol Renal Physiol 2005; 289:F31-42.

27. Uccelli A, Moretta L, Pistoia V. Mesenchymal stem cells in health and disease. Nat Rev Immunol 2008; 8:726-736. 